\title{
Protection of coronary circulation: Evaluation by PET perfusion imaging
}

\author{
Antti Saraste, MD, PhD, ${ }^{\mathrm{a}, \mathrm{b}}$ Heikki Ukkonen, $\mathrm{MD}, \mathrm{PhD},{ }^{\mathrm{b}}$ and Juhani Knuuti, \\ $M D, P h D^{a}$ \\ a Turku PET Centre, Turku University Hospital and University of Turku, Turku, Finland \\ b Heart Center, Turku University Hospital, Turku, Finland
}

Received Dec 1, 2016; accepted Dec 1, 2016

doi: $10.1007 / \mathrm{s} 12350-016-0765-\mathrm{z}$

\section{See related article, pp. 887-896}

Remote ischemic conditioning (RIC) by brief intermittent episodes of ischemia and reperfusion of an organ or tissue remote from the heart protects the myocardium against ischemia-reperfusion injury. ${ }^{1}$ Experimental studies have established RIC as a powerful cardioprotective intervention. The ability to produce the protective effect by simply inflating a blood pressure cuff placed on the upper arm or thigh inducing periods of ischemiareperfusion has been important for its translation to the clinical setting. ${ }^{1,2}$ In proof-of-concept clinical studies, RIC reduced myocardial infarct (MI) size assessed by cardiac enzymes, single-photon emission computed tomography (SPECT) perfusion imaging, or cardiac magnetic resonance (CMR) imaging by $20 \%$ to $30 \%$ when applied as an adjunct to primary percutaneous coronary intervention (PCI) or thrombolysis in patients with acute MI. ${ }^{1,3}$ The effect of RIC on long-term clinical outcomes is currently being evaluated in acute MI patients in the Effect of Remote Ischemic Conditioning Before Hospital Admission (CONDI) trial. ${ }^{4}$

The coronary vasculature has been considered as a target of RIC in addition to direct effect on the myocardium. ${ }^{5}$ Mechanisms of RIC can be briefly summarized as activation of nociceptive fibers during brief, local injury that releases an unidentified molecule into the circulation and/or signal through the spinal cord to activate both cardiac vagal and sympathetic efferent neurons to release cardioprotective substances. ${ }^{1}$ Many

Reprint requests: Antti Saraste, MD, PhD, Heart Center, Turku University hospital, Hämeentie 11, 20520 Turku, Finland; antti.saraste@utu.fi J Nucl Cardiol 2018;25:897-9.

1071-3581/\$34.00

Copyright (c) 2016 American Society of Nuclear Cardiology. endogenously derived preconditioning agents are vasoactive (e.g., adenosine, nitric oxide, and bradykinin). Thus, it has been hypothesized that RIC can improve vascular function. Studies have shown that RIC prevents peripheral endothelial dysfunction associated with ischemia, ${ }^{1,2}$ but the effects on coronary blood flow have been controversial. ${ }^{1,5}$ In an experimental model, repeated transient limb ischaemia lead to reduced coronary resistance and increased basal flow in the left anterior descending coronary artery in healthy pigs. ${ }^{6}$ Similarly, basal blood flow velocity in the left anterior descending coronary artery assessed by transthoracic Doppler echocardiography was increased shortly after RIC in healthy human subjects. ${ }^{7}$ However, similar findings were not observed in patients with ischemic heart disease undergoing PCI. ${ }^{8}$ Upper limb RIC did not reduce microvascular resistance or improve basal or hyperemic coronary flow velocity in response to ischemia-induced vasodilation when assessed by intravascular probe placed distal to a coronary stenosis. ${ }^{8}$

Positron emission tomography (PET) with radiotracer kinetic modeling can be used to quantify myocardial blood flow (MBF) in absolute terms $(\mathrm{mL} / \mathrm{g} /$ minute) at rest and during vasodilator stress that allows the computation of coronary flow reserve (CFR). Quantification of regional MBF and CFR by PET may identify microvascular dysfunction associated with early stages of atherosclerosis, better characterize the extent and severity of coronary artery disease (CAD) burden in multivessel disease, and detect balanced decreases of MBF in all major coronary artery vascular territories. ${ }^{9-12}$ Furthermore, several studies have demonstrated that reduced CFR is a powerful predictor of increased risk of future cardiac events. ${ }^{10,11}$

Quantification of MBF is accurate and technically feasible by dynamic PET with the generator-produced flow tracer ${ }^{82}$ Rubidium $\left({ }^{82} \mathrm{Rb}\right)$ that has made the technique available also at sites without an onsite 
cyclotron. ${ }^{13}$ Different software packages available can be used for analysis of global and regional MBF accurately and reproducibly. ${ }^{14,15}$ Furthermore, it has been shown that variability of rest and stress MBF in separate ${ }^{82} \mathrm{Rb}$ studies is low as shown by coefficients of repeatability of approximately $15 \%{ }^{16}$ that is an important determinant of sample size needed to detect changes in MBF. The ability to measure changes in MBF quantitatively over time makes PET perfusion imaging an attractive tool to monitor effects of therapies on myocardial perfusion and coronary vascular function. ${ }^{17}$

In current issue of the Journal, Pryds et al. report on the effect of RIC on myocardial perfusion in 49 patients with suspected ischemic heart disease. ${ }^{18}$ Perfusion abnormalities were detected by rest-stress ${ }^{82} \mathrm{Rb}$ PET in $36 \%$ (reversible in $25 \%$ ) of patients; risk factors for CAD were common and many had previously known CAD. Resting MBF was quantified by ${ }^{82} \mathrm{Rb}$ PET before and after RIC induced by 4 cycles of brief upper arm ischemia and reperfusion. Circulating levels of microribonucleic acid 144 (microRNA-144), a biomarker of RIC, were increased indicating that the procedure was effective. The results of ${ }^{82} \mathrm{Rb}$ PET showed that global resting MBF and myocardial vascular resistance were unaffected by RIC. In myocardial regions with reversible perfusion abnormality, RIC appeared to selectively reduce resting MBF. However, this effect was not present when MBF was normalized to cardiac work, a determinant of myocardial oxygen consumption. These findings argue against significant immediate effects of RIC on the amount or distribution of basal myocardial perfusion in stable patients with or at risk of CAD.

The primary goal of most of the studies applying RIC has been to provide cardioprotection against myocardial ischemia-reperfusion injury in acute MI. Evaluation of MI size and salvage (relation of MI size to the initial area at risk) by myocardial perfusion imaging is a well-validated tool for evaluation of cardioprotection. The study of Pryds et al. extends this approach by adding the quantification of regional MBF to evaluate effects of RIC on coronary vasculature in cardiac stable patients. PET imaging enables visualization of reversibly ischemic and non-ischemic myocardial areas so that therapeutic effects can be assessed separately in these regions. This is important as therapy can affect differently vascular resistance and distribution of MBF in the ischemic and non-ischemic myocardium. Some therapies may even be targeted to a specific region, such guidance of intramyocardial angiogenic gene therapy to optimal sites of reversibly ischemic and hibernating myocardium in patients with extensive CAD based on combination of quantitative PET perfusion imaging and electromechanical NOGA catheter mapping. ${ }^{19}$ The effects of therapy on myocardial perfusion can be measured by comparing changes in MBF before and after therapy. The study of Pryds et al. demonstrates how quantitative PET perfusion imaging with short lived radiotracer, such as ${ }^{82} \mathrm{Rb}$ or ${ }^{15} \mathrm{O}$-water, can be used to assess immediate effects of therapy on coronary vasculature in a carefully designed study. ${ }^{20}$ Since basal MBF is tightly coupled with myocardial work (oxygen consumption), it is important to take into account possible changes in cardiac pump function and systemic hemodynamics to be able to detect effects of therapy. Indeed, these were controlled in the study of Pryds et al. by normalizing MBF with the amount of cardiac work and rate pressure product.

Responses of MBF to vasomotor stress have been used to monitor the effects of various pharmacologic or life-style interventions on coronary vascular function. ${ }^{17,20}$ The most commonly applied approach is measurement of CFR in response to vasodilators adenosine or dipyridamole. The adenosine-induced coronary flow response reflects the combined effect of endothelium-mediated vasodilatory function and vascular smooth muscle relaxation and has been used as an integrated measure of coronary reactivity. ${ }^{17,20}$ In the study of Pryds et al., response of MBF to vasodilator stress was not studied, because the results may be confounded by adenosine and other vasoactive substances being possible mediators of RIC. Since RIC may have more prolonged effects on vascular function, ${ }^{1,21,22}$ the effects of RIC on MBF responses to vasodilator stress could be of interest in patients with stable CAD. In one study, short-term spinal cord stimulation that also modulates the nociceptive neurotransmission and the autonomic nervous system improved ischemia threshold assessed by dobutamine stress echo and adenosinestimulated MBF assessed by PET in the ischemic myocardial regions in patients with stable, refractory angina pectoris. ${ }^{23}$ In another study, RIC treatment performed twice a day for 1 week improved coronary flow velocity reserve in response to ATP-induced vasodilatation in healthy subjects and patients with reduced left ventricle systolic function. ${ }^{21}$ Whether RIC has effects on coronary reactivity in patients with stable CAD still remains to be tested.

The study of Pryds et al. concludes that RIC has no significant immediate effects on basal myocardial blood flow in patients with suspected ischemic CAD that is in line with the previous observations in patients with stable CAD. Until the endogenous effectors of RIC have been identified, it may be difficult to conclusively study the effects of RIC on coronary vascular responses to vasoactive stressors due to their potential to interfere with RIC stimulus. Thus, the primary application of RIC 
remains to confer cardioprotection against acute myocardial ischemia-reperfusion injury.

\section{Disclosures}

The authors acknowledge financial support from The Academy of Finland Centre of Excellence on Cardiovascular and Metabolic Diseases, Helsinki, Finland and Finnish Foundation for Cardiovascular Research.

\section{References}

1. Heusch G, Botker HE, Przyklenk K, Redington A, Yellon D. Remote ischemic conditioning. J Am Coll Cardiol. 2015;65:17795 .

2. Kharbanda RK, Mortensen UM, White PA, Kristiansen SB, Schmidt MR, Hoschtizky JA, et al. Transient limb ischemia induces remote ischemic preconditioning in vivo. Circulation. 2002;106:2881-3.

3. Bøtker HE, Kharbanda R, Schmidt MR, Bøttcher M, Kaltoft AK, Terkelsen CJ, et al. Remote ischaemic conditioning before hospital admission, as a complement to angioplasty, and effect on myocardial salvage in patients with acute myocardial infarction: A randomised trial. Lancet. 2010;375:727-34.

4. Hausenloy DJ, Kharbanda R, Rahbek SM, Moller UK, Ravkilde J, Okkels JL, et al. Effect of remote ischaemic conditioning on clinical outcomes in patients presenting with an ST-segment elevation myocardial infarction undergoing primary percutaneous coronary intervention. Eur Heart J. 2015;36:1846-8.

5. Heusch G. The coronary circulation as a target of cardioprotection. Circ Res. 2016;118:1643-58.

6. Shimizu M, Konstantinov IE, Kharbanda RK, Cheung MH, Redington AN. Effects of intermittent lower limb ischaemia on coronary blood flow and coronary resistance in pigs. Acta Physiol (Oxf). 2007;190:103-9.

7. Zhou K, Yang B, Zhou XM, Tan CM, Zhao Y, Huang C, et al. Effects of remote ischemic preconditioning on the flow pattern of the left anterior descending coronary artery in normal subjects. Int J Cardiol. 2007;122:250-1.

8. Hoole SP, Heck PM, White PA, Khan SN, O'Sullivan M, Clarke $\mathrm{SC}$, et al. Remote ischemic preconditioning stimulus does not reduce microvascular resistance or improve myocardial blood flow in patients undergoing elective percutaneous coronary intervention. Angiology. 2009;60:403-11.

9. Saraste A, Kajander S, Han C, Nesterov SV, Knuuti J. PET: Is myocardial flow quantification a clinical reality? J Nucl Cardiol. 2012;19:1044-59.

10. Schindler TH. Myocardial blood flow: Putting it into clinical perspective. J Nucl Cardiol. 2016;23:1056-71.
11. Johnson NP, Gould KL, Di Carli MF, Taqueti VR. Invasive FFR and noninvasive CFR in the evaluation of ischemia: What is the future? J Am Coll Cardiol. 2016;67:2772-88.

12. Kajander S, Joutsiniemi E, Saraste M, Pietilä M, Ukkonen H, Saraste A, et al. Cardiac positron emission tomography/computed tomography imaging accurately detects anatomically and functionally significant coronary artery disease. Circulation. 2010;122:603-13.

13. Moody JB, Lee BC, Corbett JR, Ficaro EP, Murthy VL. Precision and accuracy of clinical quantification of myocardial blood flow by dynamic PET: A technical perspective. J Nucl Cardiol. 2015;22:935-51.

14. Nesterov SV, Deshayes E, Sciagrà R, Settimo L, Declerck JM, Pan $\mathrm{XB}$, et al. Quantification of myocardial blood flow in absolute terms using (82)Rb PET imaging: The RUBY-10 study. JACC Cardiovasc Imaging. 2014;7:1119-27.

15. Dunet V, Klein R, Allenbach G, Renaud J, deKemp RA, Prior JO. Myocardial blood flow quantification by Rb-82 cardiac PET/CT: A detailed reproducibility study between two semi-automatic analysis programs. J Nucl Cardiol. 2016;23:499-510.

16. El Fakhri G, Kardan A, Sitek A, Dorbala S, Abi-Hatem N, Lahoud $\mathrm{Y}$, et al. Reproducibility and accuracy of quantitative myocardial blood flow assessment with (82)Rb PET: Comparison with (13)Nammonia PET. J Nucl Med. 2009;50:1062-71.

17. Schindler TH, Schelbert HR, Quercioli A, Dilsizian V. Cardiac PET imaging for the detection and monitoring of coronary artery disease and microvascular health. JACC Cardiovasc Imaging. 2010;3:623-40

18. Pryds K, Nielsen RR, Hoff CM, Tolbod LP, Bouchelouche K, Li J, et al. Effect of remote ischemic conditioning on myocardial perfusion in patients with suspected ischemic coronary artery disease. J Nucl Cardiol. 2016. doi:10.1007/s12350-016-0709-7.

19. Hassinen I, Kivelä A, Hedman A, Saraste A, Knuuti J, Hartikainen $\mathrm{JE}$, et al. Intramyocardial gene therapy directed to hibernating heart muscle using a combination of electromechanical mapping and positron emission tomography. Hum Gene Ther. 2016. doi: 10.1089/hum.2016.131.

20. Johansson BL, Sundell J, Ekberg K, Jonsson C, Seppänen M, Raitakari O, et al. C-peptide improves adenosine-induced myocardial vasodilation in type 1 diabetes patients. Am J Physiol Endocrinol Metab. 2004;286:E14-9.

21. Kono Y, Fukuda S, Hanatani A, Nakanishi K, Otsuka K, Taguchi $\mathrm{H}$, et al. Remote ischemic conditioning improves coronary microcirculation in healthy subjects and patients with heart failure. Drug Des Devel Ther. 2014;8:1175-81.

22. Loukogeorgakis SP, Panagiotidou AT, Broadhead MW, Donald A, Deanfield JE, MacAllister RJ. Remote ischemic preconditioning provides early and late protection against endothelial ischemiareperfusion injury in humans: Role of the autonomic nervous system. J Am Coll Cardiol. 2005;46:450-6.

23. Saraste A, Ukkonen H, Varis A, Vasankari T, Tunturi S, Taittonen $\mathrm{M}$, et al. Effect of spinal cord stimulation on myocardial perfusion reserve in patients with refractory angina pectoris. Eur Heart $\mathbf{J}$ Cardiovasc Imaging. 2015;16:449-55. 